\title{
Metodología de negociación abierta y su eficacia en el ámbito mercantil de Ecuador
}

\section{Open negotiation methodology and its effectiveness in the commercial field of Ecuador}

Isidro Fierro Ulloa ${ }^{a}$, L. Giovanny Veintimilla Vélez ${ }^{b}$

\author{
INFORMACIÓN DEL \\ ARTÍCULO \\ Fecha de recepción: 18 de julio de \\ 2017 \\ Fecha de aceptación: 21 de septiembre \\ de 2017
}

a MBA, Universidad Santa María. Decano de la Facultad de Estudios Internacionales de la UEES-Ecuador. E-mail:isfierro@uees.edu.ec

${ }^{b}$ MAE, UEES-Ecuador. Ingeniero Comercial, Pontificia Universidad Católica del Ecuador. E-mail: lveintimilla@uees.edu.ec

\begin{abstract}
Resumen
El procedimiento de Negociación Abierta procura encaminar a las partes hacia acuerdos viables, con base en la transparencia de la información. Con el fin de someter a demostración esta hipótesis, se llevó a cabo una prueba de laboratorio, en la que un grupo de encuestados fue sometido a dos escenarios, uno que sirvió de control y otro sobre el cual operaron las variables de interés experimental.Los resultados de la investigación fueron concluyentes respecto a la aplicación de un modelo predictivo en los procesos de negociación, cuyo teorema es el siguiente: las partes llegarán a un acuerdo solo después de haber alcanzado el punto de convergencia de sus costos de oportunidad. Además, se encontró un axioma contra-factual de la Negociación Abierta: los procesos de negociación en los que las partes no aportan información pertinente y relevante, respecto de su situación y expectativas, o especulan con ésta, no llegan a ser viables, beneficiosos ni sostenibles. Según este nuevo paradigma, es más provechoso informar, en lugar de suponer, ocultar o especular.
\end{abstract}

Palabras Clave:

Expectativas, costos de oportunidad, costos marginales, información, negociación abierta.

Clasificación JEL: M0. 


\section{Metodología de negociación abierta y su eficacia en el ámbito mercantil de Ecuador}

\section{Open negotiation methodology and its effectiveness in the commercial field of Ecuador}

Isidro Fierro Ulloa ${ }^{a}$, L. Giovanny Veintimilla Vélez ${ }^{b}$

\author{
INFORMACIÓN \\ DEL \\ ARTÍCULO \\ Fecha de recepción: 18 de julio de \\ 2017 \\ Fecha de aceptación: 21 de septiembre \\ de 2017
}

a MBA, Universidad Santa María. Decano de la Facultad de Estudios Internacionales de la UEES-Ecuador. E-mail:isfierro@uees.edu.ec.

${ }^{b}$ MAE, UEES-Ecuador. Ingeniero Comercial, Pontificia Universidad Católica del Ecuador. E-mail: lveintimilla@uees.edu.ec

\begin{abstract}
The open negotiation procedure seeks to direct the parties towards viable agreements, based on the transparency of the information. In order to test this hypothesis, a laboratory test was carried out, in which a group of respondents was subjected to two scenarios, one that served as control and the other on which variables of experimental interest operated. The results of the investigation were conclusive regarding the application of a predictive model in the negotiation processes, whose theorem is as follows: the parties will reach an agreement only after having reached the point of convergence of their opportunity costs. In addition, a counter-factual axiom of the open negotiation was found: the negotiation processes in which the parties do not provide relevant and relevant information, regarding their situation and expectations, or speculate with it, do not become viable, beneficial or sustainable. According to this new paradigm, it is more profitable to inform, rather than suppose, to hide or speculate.
\end{abstract}

\section{Keywords}

Expectations, opportunity costs, marginal cost, information, open negotiation.

JEL Classification: M0. 


\section{Introducción}

En toda dinámica empresarial se persiguen acuerdos transaccionales, en los que las partes se comprometan a intercambiar bienes o servicios de modo equitativo. Con el nuevo paradigma ganar-ganar propuesto por Fisher, Ury, y Patton (1979), la teoría de la negociación evolucionó desde el objetivo unilateral de apropiarse de los mayores beneficios, hacia identificar los medios de expandirlos, descubriendo y conciliando intereses bilaterales. Una nueva perspectiva de la negociación, como arte y ciencia, la ofreció Raiffa (1982) quien trascendió la clásica dualidad de ganar o perder, hacia encontrar la fórmula de ampliar y luego negociar la división de los beneficios. Más tarde, Lax, y Sebenius (1986) brindaron una nueva visión acerca de las dinámicas de negociación cooperativas que crean valor, en contraste con las tensiones competitivas que intervienen para reclamarlo. En la siguiente década de los 90, la investigación sobre la negociación, integró el estudio empírico de cómo la gente negocia realmente, con los conceptos de la teoría de juegos bajo el supuesto de racionalidad (Bazerman y Neale, 1992). En todo sentido es de suma importancia que el tema de la negociación y su respectiva preparación deban ser tomados de forma muy profesional (Henao, Fierro, y Cardona, 2017).

Hoy en día, la teoría vigente distingue dos métodos básicos de negociación. Uno con ánimo distributivo y competitivo, en el que sólo puede haber un ganador, cuyos beneficios los obtendrá a expensas de su contraparte, es decir, un juego de suma cero (Mehta, 2012). Y otro método, doctrinalmente opuesto al primero, que viene inspirado en los principios de integración y cooperación, en el que de modo intencional las partes buscan las opciones que más les beneficien recíprocamente, sin que nadie resulte perdedor (Mullender, 2014).

Mientras el modelo distributivo se concentra en una sola variable - por ejemplo, el precio- el procedimiento integrador y cooperativo procura contextualizar la negociación en un marco más amplio, incorporando variables adicionales de mutuo interés (Mehta, 2012). Como se ha mencionado, el método integrativo consiste en incorporar aspectos conexos a la materia de negociación, siempre que éstos puedan configurar un paquete de beneficios para ambas partes; y de esta forma, los elementos más sensibles podrían ser balanceados y compensados con las demás variables incorporadas (Sebenius, 2001).

El problema que motiva el presente artículo es la omisión funcional y metodológica que actualmente presenta la teoría de la negociación: la tesis académica dominante ha puesto énfasis en las formalidades conductuales, dejando de lado el examen de la información situacional de las partes. El error de valoración que generalmente afecta a las negociaciones o gestión de conflictos, consiste en discutir alrededor de elementos totalizadores - agregados o acumulados - en lugar de considerar sus variaciones marginales. Definiéndolo de modo conciso, el valor marginal es la variación en el beneficio o en el costo total, que trae consigo la 
última unidad adicional producida $\mathrm{o}$ consumida. Así, la actividad económica, y la vida misma, están sometidas a un ciclo de desarrollo, en el cual ni los beneficios ni los costos mantienen constante su tasa de crecimiento, conforme aumenta el volumen de transacciones o el consumo de recursos. De hecho, los beneficios tienden a desacelerar sus valores incrementales —crecen cada vez en menor medida o incluso decrecen-, en tanto que, los costos tienden a aumentar progresivamente cada vez en mayor medida. Tal comportamiento se da por efecto de la ley de los rendimientos marginales decrecientes, que opera no solo en materia económica, sino también en todos los ámbitos de la actividad humana.

En tal contexto, la propuesta central del presente artículo recomienda que las partes contrasten con honestidad, desde un inicio, tanto los beneficios como los costos marginales vinculados con la situación en conflicto, o los que se derivarán de las transacciones que proyecten llevar a cabo entre sí. Bajo estas reglas de transparencia, cada actor lograría una comprensión cabal de los beneficios y costos de su contraparte, con lo cual, ambas partes podrían acordar los términos que consideren más balanceados. Así, siempre sería posible arribar a un acuerdo, si las partes valorasen los costos de su (des)acuerdo.

Por tanto, se propone demostrar la siguiente hipótesis: los acuerdos equitativos y sostenibles se derivan de procesos de Negociación Abierta -Open Negotiation- en los que desde su inicio, cada una de las partes revela información pertinen- te y relevante, respecto de su situación y expectativas.

Esta propuesta encuentra su justificación en razón de que los intercambios se dan en todos los ámbitos de la vida. Los acuerdos y sus fórmulas de negociación viabilizan tales intercambios, sin los cuales, sería imposible cimentar relaciones económicas y edificar sistemas de convivencia social. De allí su importancia vital y la necesidad de estudiarlos, a fin de comprender la interacción de sus variables, y llegar al diseño de un modelo eficaz de negociación y de resolución de conflictos.

\section{Metodología}

Las investigaciones realizadas por Weingart, Olekalns, y Smith (2004), les llevó a concluir que el primer paso en el estudio de los procesos de negociación es determinar qué patrones de comportamiento son relevantes. Enfatizan que este primer paso tiene una profunda incidencia en la formulación y prueba de hipótesis. A modo de ejemplo $-\mathrm{y}$ en directa relación con el tema del presente artículo- los mencionados académicos reportan que en sus primeras investigaciones, encontraron que no había una clara relación causal entre el intercambio de información y la calidad de los acuerdos (Weingart, Thompson, Bazerman, y Carroll, 1990). Sin embargo, en posteriores investigaciones demostraron que aquello se debía a que no se habían distinguido los diferentes tipos de información. En efecto, el intercambio de información prioritaria para los actores, influía positivamente en la calidad de sus acuerdos, mientras que la 
información sobre sus preferencias y posiciones, influía negativamente en la calidad de los acuerdos (Hyder, Prietula, y Weingart, 2000; Olekalns y Smith, 2000; Olekalns, Smith, y Walsh, 1996; Weingart, Hyder, y Prietula, 1996).

De otra parte, Pruitt (2005) menciona que en los estudios experimentales cuyo diseño incluye la manipulación de variables, es factible distinguir el efecto y su causa. Esquematiza cuatro posibles explicaciones, cuando un estudio sugiere una relación o covariación entre dos variables: $X$ tiene un efecto causal sobre $Y ; Y$ influye en $X$; un tercer factor común $Z$, influye tanto en $X$ como en $Y$, lo que produce una falsa relación entre estas dos variables; $y$ por último, la relación entre $X \mathrm{y} Y$ es debida al azar.

Las pruebas estadísticas de significación -dado un nivel de confianzapermiten descartar la explicación de que la relación entre $X$ y $Y$ sea aleatoria. Si el estudio es un experimento y $X$ es una variable manipulada, por lo general, se puede descartar que $Y$ incide en $X$, o la existencia de un tercer factor común $Z$, con el argumento de que el investigador es la única fuente de variación en $\mathrm{X}$; y por tanto, ni $Y$ ni $Z$ pueden haber influido en aquella variable. Este razonamiento desprende la única explicación plausible de que, en efecto, $X$ influye directamente sobre $Y$ (Pruitt, 2005).

Bajo ciertas condiciones, aunque el laboratorio difiere en muchas maneras del estudio de campo, generalmente no afecta la capacidad de generalizar. Sin embargo, se debe tener especial cuidado en identificar si las manipulaciones efectuadas en el laboratorio, no han producido procesos psicológicos o sociales atípicos que afecten a las variables dependientes. De ser así, los resultados que se obtengan en el laboratorio no coincidirán necesariamente con los que se produzcan en los entornos de la vida real.

En el marco de la presente investigación, en principio se consideró efectuar una prueba de laboratorio, en la que interactuaran pares de negociadores, bajo ciertas reglas de juego. No obstante, se presentaron dificultades en convocar y reunir un número suficiente de participantes. Por otra parte, como alternativa, el levantamiento de datos de campo únicamente mediante encuestas, no aportaría una sólida comprobación de la hipótesis de investigación.

De este modo, se diseñó un cuestionario con el fin de observar las decisiones de los participantes frente a dos entornos de negociación. A diferencia del método habitualmente seguido en las pruebas experimentales, el grupo de encuestados fue único y se lo sometió a dos escenarios, uno que sirvió de control y otro que cumplió el objetivo experimental. El grupo de encuestados respondió a la siguiente caracterización: género (30\% hombres, $70 \%$ mujeres); franja etaria (22 a 38 años); actividad laboral (fuerza de ventas de seguros de vida y medicina de prepago); ciudad (Quito). El cuestionario fue ubicado en una plataforma web, a la cual accedieron individualmente cada uno de los encuestados, con libertad de horario.

La Tabla 1 reproduce exactamente el primer cuadro de instrucciones que tuvo que leer cada persona encuestada. 
Tabla 1.

Primer cuadro de instrucciones

(SERÁN DOS JUEGOS)

PRIMER DESAFÍO: Tal vez abandone.

- Intentaré comprarte una obra de arte.

- El precio de esa obra no es fijo, yo te propondré el precio.

- Cada vez que te proponga un precio, podrás aceptarlo o no.

- Si no aceptas mi precio tal vez abandone, sin comprar tu obra de arte.

Fuente: Elaboración del autor

Cumplieron un propósito definido que se explica a continuación:

- Intentaré comprarte una obra de arte. De inicio asigna a la persona encuestada el rol de vendedor. La precisión de que se trata de una obra de arte, determina que es un artículo único, disponible para un solo comprador, sin copias adicionales ni reproducciones en serie. La alusión a obra de arte, no sugiere que su precio tenga que ser elevado.

- El precio de esa obra no es fijo, yo te propondré el precio. Esclarece el objetivo del juego, es decir, una libre interacción entre el encuestado vendedor y el sistema informático que asume el rol de interesado comprador. Esclarece, además, que las propuestas de precio las hará el interesado comprador.

- Si no aceptas mi precio tal vez abandone, sin comprar tu obra de arte. Esta instrucción introduce un elemento de incertidumbre, en relación a la cantidad de propuestas que hará el interesado comprador. Por tanto, se desconoce cuál será el umbral hasta el cual podrán llegar los precios propuestos.

En línea con el objetivo de este primer juego de decisión - cumplir la función de escenario de control- intencionalmente se evitó informar al vendedor encuestado, acerca de si hay o no otros interesados en la misma obra. Este dato se dejó difuso con el fin de que el encuestado adoptara libremente cualquier suposición al respecto. La sintaxis y pronombres utilizados en las instrucciones, tuvieron la intención de establecer una relación personalizada y de inmediata confianza con el encuestado. El uso del término desafio tuvo la finalidad de provocar en la persona encuestada, el compromiso necesario para personificar el rol asignado y concluir la prueba experimental. Asimismo, se consideró prioritario que desde un primer instante el encuestado entendiera el rol que asumiría durante la prueba experimental.

Luego de leer estas instrucciones, se abrió ante el encuestado la primera propuesta de precio, tal como se ilustra en la Tabla 2. 
Tabla 2.

Cuadro de decisión

\begin{tabular}{c|cc}
\hline Hora & \multicolumn{2}{|c}{ Tal vez esta sea mi última propuesta } \\
\hline 8:00 AM & $\$ 1.000$ \\
\hline & ¿Aceptas mi propuesta? \\
& $\square$ No & $\square$ Sí \\
\hline
\end{tabular}

Fuente: Elaboración del autor

Como se puede apreciar, cada cuadro de propuesta advertía acerca de la posibilidad de que tal vez sea la última oferta, con lo cual se obligaba al vendedor encuestado a ponderar su propia aversión o propensión a tomar riesgos. Es decir, se trataba de una elección con las siguientes implicaciones: aceptar el precio propuesto, admitiendo la posibilidad de que podría haber logrado un precio mayor; o, rechazar el precio propuesto, aceptando el riesgo de no recibir más ofertas del interesado, y que éste se marche sin comprar la obra. En caso de optar por la respuesta negativa, a continuación se le presentaba al encuestado un nuevo cuadro con variantes en la hora y el precio. La hora fue progresando en intervalos de cinco minutos, y el precio fue escalando, aunque a una tasa marginalmente decreciente, hasta llegar finalmente a $\$ 4.000$ a las 10:00AM -en total fueron veinticinco propuestas-

En caso de haber respondido negativamente también a esta última propuesta - el encuestado desconoce que no habrá otra adicional- seguidamente se expuso el texto que consta en la Tabla 3. Con este cuadro final, o con una respuesta afirmativa a cualquiera de las propuestas anteriores, se procedió a cerrar el primer escenario de juego.

Tabla 3.

Mensaje final del primer desafio

Lo lamento, ya no te haré más propuestas, me marcho sin comprar tu obra de arte.

Fuente: Elaboración del autor

Inmediatamente después se abrió un nuevo cuadro de instrucciones para el segundo escenario, tal como detalla en la Tabla 4.

Tabla 4.

Segundo cuadro de instrucciones

SEGUNDO DESAFÍO: ¿Me esperarías?

- Intentaré comprarte una obra de arte.

- El precio de esa obra no es fijo, yo te propondré el precio.

- Como verás, según pasen los minutos, mejoraré mi precio pero disminuirá la cantidad de interesados esperando por la misma obra.

- En la siguiente tabla, elige hasta qué hora me esperarías.

Fuente: Elaboración del autor 
De modo análogo al primer escenario, cada una de las instrucciones del segundo desafío cumplió un propósito específico e intencionado. El segundo juego introdujo las siguientes variables independientes de experimentación: exhibición de la información completa acerca de las expectativas del comprador, expresadas en los precios que estaría dispuesto a ofertar de modo sucesivo, y el tiempo que dedicaría, incluyendo el umbral máximo hasta el cual llegaría su propuesta de precio y en qué momento; exhibición de la información completa acerca de la pérdida de recursos del vendedor, expresada en la reducción de su cartera de personas interesadas en la misma obra de arte; y, eliminación de la incertidumbre respecto a las intenciones, beneficios y costos marginales del comprador y vendedor.

El precio creció de modo marginalmente decreciente, en contraste con la cantidad de interesados cuyo descenso es acelerado. De este modo, este segundo escenario incorporó dos elementos esenciales del teorema de Negociación Abierta: información completa acerca de las expectativas del comprador, rol que asumió el sistema informático; e, información completa acerca de los beneficios y costos marginales del tomador de decisión, en este caso, el encuestado que asumió el rol de vendedor.

Bajo estas condiciones de Negociación Abierta debería esperarse, por tanto, que las elecciones de precio sean de mejor calidad - mayor valor monetario- que las correspondientes al primer escenario.

En la Figura 1, la curva ascendente representa el costo marginal del vendedor de la obra de arte - persona que se sometió a la prueba experimental - trazada en función del tiempo que ha acumulado en negociar con el posible comprador. Como se puede observar, su costo marginal crece a medida que consume más tiempo en considerar las propuestas del comprador, pues mientras no decida aceptar el precio que aquel le ofrece, seguirá acumulando pérdidas por el abandono - cada vez más acelerado- de otros interesados que estaban esperando la ocasión de presentar sus ofertas de precio. La gráfica ilustra el costo marginal del vendedor, en su tramo ascendente, pues únicamente en este segmento tendrá el incentivo para aceptar uno de los precios propuestos por el interesado, ya que el abandono de más interesados constituye una pérdida de recursos para el vendedor. De la misma forma, describe la curva de costo marginal de oportunidad del vendedor, también como una función del tiempo que éste ha invertido en esperar una mejor propuesta de precio de parte del primer interesado. Esta función desciende, pues a medida que transcurre más tiempo, sin tomar una decisión de aceptación, las propuestas subsiguientes tienen un menor valor incremental.

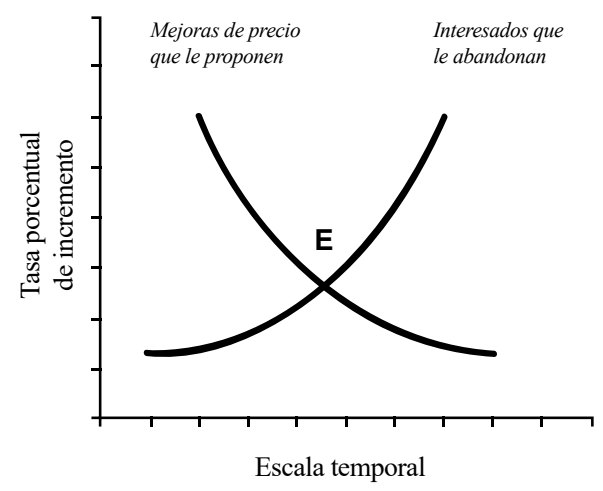

Figura 1. Prueba experimental: Costos marginales del vendedor (tomador de decisión).

Fuente: Elaboración del autor 
En consecuencia, tal vendedor irá moderando a la baja sus expectativas de alcanzar un mejor precio, y tomará consciencia acerca de los beneficios que se abstiene de percibir por su negativa a aceptar un precio - costos de oportunidad-.

El balance de intereses -en este caso, los que pertenecen al mismo vendedorviene marcado por el punto de equilibrio $E$, que representa la igualdad de sus costos marginales, tanto los de oportunidad que descienden, como los de pérdidas de recursos que ascienden.

Se debe anotar que esta prueba experimental se ha llevado a cabo, únicamente desde la perspectiva unilateral del vendedor, como tomador de decisiones, puesto que la negociación es un fenómeno diádico, el contexto ideal de una experimentación debería considerar también los costos marginales que debe soportar el comprador, es decir, las oportunidades y recursos que éste va sacrificando cada vez que se ve obligado a ofertar un mejor precio.

En tal sentido y para efectos de la presente prueba experimental, es válido entonces considerar la siguiente suposición técnica: el comprador, cuyo rol ha sido asumido de modo virtual por el sistema informático, realiza las propuestas de precio como resultado de sus propias expectativas, costos de oportunidad y consumo de recursos, cuyas funciones marginales las conoce con exactitud, y se consideran estables dentro del contexto de la prueba experimental.

El punto de equilibrio $E$ determinará el precio que finalmente decidirá aceptar el vendedor, dentro de un proceso de Negociación Abierta, en el que tanto comprador como vendedor compartieron información respecto a la variación marginal de precios y costos.

Según la percepción del vendedor, a la izquierda de $E$ sus costos de oportunidad -incrementos de precio propuestos por el posible comprador - todavía crecen a una tasa superior que la de sus pérdidas. En tanto que, a la derecha de $E$, las pérdidas del mismo vendedor - cantidad de interesados que dejan de esperar- crecen a una tasa superior que la de sus costos de oportunidad.

Por consiguiente, el punto de equilibrio $E$ marca la igualdad de las tasas de variación porcentual de ambas magnitudes, donde el vendedor no sacrificará un valor porcentual mayor ni menor de ambas variables, y por tanto, constituye su propio nivel de equidad u óptimo de decisión.

En un procedimiento de Negociación Abierta, el vendedor debería converger - racional o intuitivamente- hacia el nivel de equilibrio $E$, y aceptar allí el precio propuesto por el comprador, pues no deseará deteriorar aún más su situación ni seguir perdiendo la oportunidad de mejorarla.

La evolución de sus costos marginales ofrece al tomador de decisión —en la presente prueba, el vendedor encuestadoel incentivo para aceptar un acuerdo. Por un lado, el vendedor tendrá una mayor propensión a transigir en el precio propuesto por el cliente, pues deseará contener sus 
pérdidas de recursos —abandonos de otras personas que inicialmente tuvieron interés por adquirir la misma obra de arte-; y por otro lado, el mismo vendedor aumentará su disposición a aceptar un precio antes de extinguir totalmente sus oportunidades de venta, puesto que las mejoras en el precio cada vez serán de menor cuantía.

\section{Resultados}

Como se puede distinguir en la Tabla 5 , hubieron encuestados que no lograron exponerse a ambos escenarios: 37 y 38 , sólo se sometieron a la prueba de control o a la experimental, respectivamente.

Tabla 5.

Grupo objetivo

\begin{tabular}{c|c|c}
\hline \multicolumn{3}{c}{ CANTIDAD DE PERSONAS QUE SE SOMETIERON A LA PRUEBA EXPERIMENTAL } \\
\hline Escenario de control & Escenario experimental & Ambos escenarios \\
\hline 37 & 38 & 33 \\
\hline
\end{tabular}

Fuente: Elaboración del autor

En consecuencia, la tabulación válida de resultados, únicamente debió referirse al grupo de 33 personas, cuyas decisiones se pudieron contrastar en los dos escenarios.

De este modo, la Tabla 6 cuantifica la cantidad de encuestados ni que bajo el rol de vendedores, decidieron aceptar uno de los precios. Y en cada caso, en la columna contigua se ha calculado su frecuencia relativa $f i$. Desde luego, la última fila resume las sumatorias de $\sum n i$ y $\sum f i$, que corresponden a la totalidad de encuestados $N i$ y al cien por ciento de frecuencias $\mathrm{Fi}$ para ambos escenarios.

Tabla 6.

Concentración de resultados

\begin{tabular}{c|c|c|c|c|c}
\hline \multirow{2}{*}{$\begin{array}{c}\text { Franja } \\
\text { horaria }\end{array}$} & \multirow{2}{*}{$\begin{array}{c}\text { Franja de } \\
\text { precios } \$\end{array}$} & \multicolumn{4}{|c}{ ESCENARIOS } \\
\cline { 3 - 6 } & & \multicolumn{2}{c|}{ CONTROL } & \multicolumn{2}{c}{ EXPERIMENTAL } \\
\cline { 3 - 6 } & $\mathrm{n}_{\mathrm{i}}$ & $\mathrm{f}_{\mathrm{i}}$ & $\mathrm{n}_{\mathrm{i}}$ & $\mathrm{f}_{\mathrm{i}}$ \\
\hline $8: 00$ a $8: 20$ & 1.000 a 1.900 & 19 & 0,58 & 7 & 0,21 \\
\hline $8: 25$ a $8: 45$ & 2.100 a 2.800 & 7 & 0,21 & 4 & 0,12 \\
\hline $8: 50$ a $9: 10$ & 2.950 a 3.450 & 3 & 0,09 & 3 & 0,09 \\
\hline $9: 15$ a $9: 35$ & 3.550 a 3.850 & & & 4 & 0,12 \\
\hline 9:40 a $10: 00$ & 3.900 a 4.000 & 4 & 0,12 & 15 & 0,46 \\
\hline \multicolumn{2}{c}{$\Sigma$} & 33 & 1 & 33 & 1 \\
\hline
\end{tabular}

Fuente: Elaboración del autor 
Es evidente que la prueba de control y la experimental arrojaron perfiles opuestos de distribución de frecuencias. El escenario de control registró la más alta concentración en el primer intervalo de precios $(58 \%)$, en tanto que en el escenario experimental el mayor conglomerado correspondió a la última franja de precios (46\%).

Tabla 7.

Estadísticos de la prueba experimental

\begin{tabular}{c|c|c|c}
\hline \multirow{2}{*}{ Estadístico } & \multirow{2}{*}{ Simbología } & \multicolumn{2}{|c}{ ESCENARIOS } \\
\cline { 3 - 4 } & & CONTROL & EXPERIMENTAL \\
\cline { 3 - 4 } & & valor & valor \\
\hline Tamaño de muestra & $\mathrm{n}$ & & 33 \\
\hline Sumatoria de precios & $\sum \mathrm{x}_{\mathrm{i}} \mathrm{n}_{\mathrm{i}}$ & $\$ 65.610$ & $\$ 101.550$ \\
\hline Valor medio & $\overline{\mathrm{x}}$ & $\$ 1.988,18$ & $\$ 3.077,27$ \\
\hline Desviación estándar & $\mathrm{S}_{\mathrm{x}}$ & $\$ 1.040,13$ & $\$ 1.183,55$ \\
\hline Mediana & $\mathrm{M}_{\mathrm{e}}$ & $\$ 1.690$ & $\$ 3.850$ \\
\hline Moda & $\mathrm{M}_{\mathrm{o}}$ & $\$ 1.000$ & $\$ 4.000$ \\
\hline
\end{tabular}

Fuente: Elaboración del autor

Tabla 8.

Prueba de significación de medias

\begin{tabular}{c|c|c}
\hline Estadístico & Simbología & Valor \\
\hline Valor medio propuesto & $\mu_{0}$ & $\$ 1.988,18$ \\
\hline Valor medio de la muestra & $\overline{\mathrm{x}}$ & $\$ 3.077,27$ \\
\hline Desviación estándar de la muestra & $\mathrm{S}_{\mathrm{x}}$ & $\$ 1.183,55$ \\
\hline Hipótesis nula & $\mathrm{H}_{0}$ & $\mu=1.988,18$ \\
\hline Hipótesis alternativa & $\mathrm{H}_{1}$ & $\mu>1.988,18$ \\
\hline Nivel de significación & $\alpha$ & 0,0005 \\
\hline Grados de libertad & $\mathrm{v}=\mathrm{n}-1$ & 32 \\
\hline Valor crítico, distribución t-Student & $\mathrm{t}$ & 3,622 \\
\hline Valor estadístico de prueba $\mathrm{t}$ & $\mathrm{t}_{\text {prueba }}=\frac{\mathrm{x}-\mu_{0}}{\mathrm{~S}_{\mathrm{x}} / \sqrt{\mathrm{n}}}$ & 5,286 \\
\hline
\end{tabular}

Fuente: Elaboración del autor

Con el fin de evaluar si tal diferencia en los resultados se podría atribuir a factores aleatorios o de simple casualidad, se deben ensayar pruebas de significación estadística. A tal propósito, las Tablas 7, 8, y 9, resumen los estadísticos de ambos escenarios. 
Tabla 9.

Prueba de signos y rangos jerarquizados

\begin{tabular}{|c|c|c|}
\hline Estadístico & Simbología & Valor \\
\hline Pares de observaciones & $\mathrm{N}$ & 21 \\
\hline Suma de rangos observada & $\mathrm{T}$ & 244 \\
\hline Suma esperada de rangos & $\mathrm{U}_{\mathrm{t}}=\frac{1}{2}\left[\frac{\mathrm{N}(\mathrm{N}+1)}{2}\right]$ & 115,50 \\
\hline $\begin{array}{l}\text { Desviación estándar } \\
\text { (distribución normal) }\end{array}$ & $\begin{array}{l}\sigma_{\mathrm{t}} \\
=\sqrt{\frac{\mathrm{N}(\mathrm{N}+1)(2 \mathrm{~N}+1)}{24}}\end{array}$ & 28,77 \\
\hline Hipótesis nula & $\mathrm{H}_{0}$ & Ausencia de efecto \\
\hline Hipótesis alternativa & $\mathrm{H}_{1}$ & $\begin{array}{c}\text { Evidencia de } \\
\text { efecto no casual }\end{array}$ \\
\hline Nivel de significación & $\alpha$ & 0,0001 \\
\hline Valor crítico & $\mathrm{Z}$ & 3,62 \\
\hline Valor estadístico de prueba & $\mathrm{z}_{\mathrm{t}}=\frac{\mathrm{T}-\mathrm{U}_{\mathrm{t}}}{\sigma_{\mathrm{t}}}$ & 4,47 \\
\hline
\end{tabular}

Fuente: Elaboración del autor

En consecuencia, se debe rechazar la hipótesis nula $H_{0}$ y aceptar la hipótesis alternativa $H_{l}$. Esto significa que tanto la distribución de frecuencias, como la tendencia central y la dispersión del escenario experimental, no son efecto de la casualidad, sino de la incidencia de condiciones diferenciadoras que operaron sobre tal escenario.

\section{Discusión}

En la Figura 2 se ilustra la distribución de frecuencias para cinco intervalos de precio. Puede constatarse que ambos escenarios describen un patrón de concentración hacia los extremos y que declina hacia el centro. Así, el ensayo de control presenta un marcado sesgo hacia la izquierda, altamente concentrado en la franja inicial de precios. En contraste con el escenario experimental, en el que la mayor proporción de decisiones correspondió al intervalo final de precios. Ambos grupos también exhiben una pauta general de descenso hacia el centro, donde la cantidad de elecciones fueron mínimas. Se destaca el hecho de que en los dos escenarios se registraron idéntica cantidad de elecciones dentro del tercer intervalo de clase. Inclusive, en el cuarto segmento de precios, el escenario de control no produjo ninguna elección. A diferencia de la situación experimental, en la que se permitió al tomador de decisión valorar las expectativas y costos de oportunidad, y con ello progresó hacia niveles más altos de precio. 


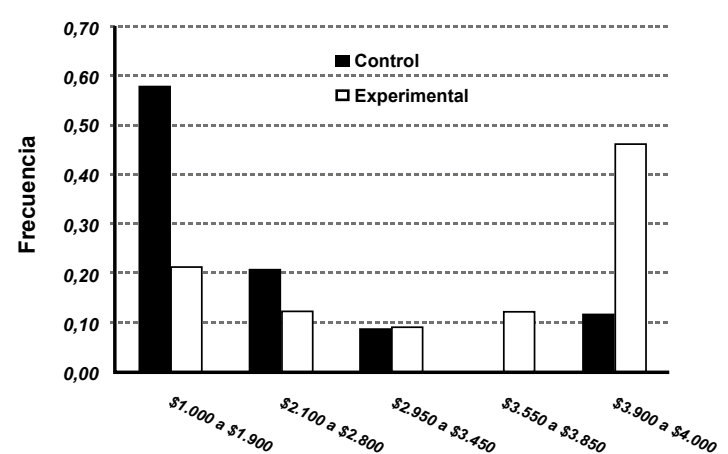

Figura 2. Distribución de decisiones en los dos escenarios.

Fuente: Elaboración del autor

Por su parte, la Figura 3 también ilustra las curvas de variación marginal - porcentual- de los costos del vendedor, y su punto de convergencia $E$ donde era previsible encontrar la mayor proporción de aceptaciones de precio. Debe recordarse que los costos se refieren a las pérdidas de oportunidad por la expectativa de lograr un mejor precio, así como los costos implícitos por la reducción de la cartera de posibles compradores. Según el postulado teórico, los acuerdos de precio en el escenario experimental, debieron concentrarse alrededor de tal intersección $E$, esto es, ligeramente por encima del $5 \%$ de ambas curvas marginales, según la apreciación visual.

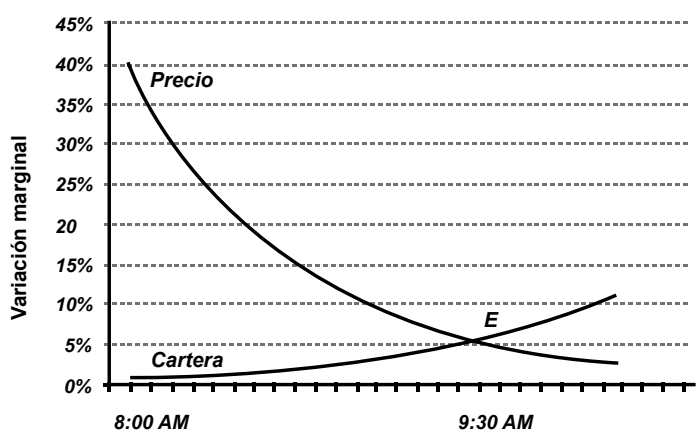

Figura 3. Punto de convergencia de variación de costos marginales.

Fuente: Elaboración del autor

La Tabla 10 contiene el cómputo detallado de las variaciones marginales, tanto de precio como de cartera, expresadas en valores absolutos y porcentuales. Valores que han sido utilizados en la confección de la Figura 2 en la que se ha ilustrado la trayectoria de las variaciones marginales y su punto de intersección. De acuerdo con esta tabla, la coincidencia porcentual -entre $6 \%$ y $6,3 \%$ - correspondió al precio de $\$ 3.850$, y por tanto, en este nivel debía darse la mayor cantidad de decisiones. Aunque la distribución de frecuencias del escenario experimental, no coincidió exactamente con tal estimación puntual, a partir del referido precio se ha concentrado la mayor proporción de decisiones. 
Tabla 10.

Variaciones marginales

\begin{tabular}{|c|c|c|c|c|c|c|}
\hline \multirow[t]{2}{*}{ H:MM } & \multicolumn{2}{|c|}{$\begin{array}{c}\text { VARIACION } \\
\text { MARGINAL } \\
\text { PRECIO }\end{array}$} & \multirow[t]{2}{*}{ Precio \$ } & \multirow[t]{2}{*}{ Cartera } & \multicolumn{2}{|c|}{\begin{tabular}{|c} 
VARIACION \\
MARGINAL \\
CARTERA
\end{tabular}} \\
\hline & $\%$ & $\Delta$ & & & $\Delta$ & $\%$ \\
\hline $8: 00$ & & & 1.000 & 300 & 0 & \\
\hline $8: 05$ & $24,0 \%$ & 240 & 1.240 & 299 & 1 & $0,3 \%$ \\
\hline $8: 10$ & $23,0 \%$ & 230 & 1.470 & 297 & 2 & $0,7 \%$ \\
\hline $8: 15$ & $22,0 \%$ & 220 & 1.690 & 294 & 3 & $1,0 \%$ \\
\hline $8: 20$ & $21,0 \%$ & 210 & 1.900 & 290 & 4 & $1,3 \%$ \\
\hline $8: 25$ & $20,0 \%$ & 200 & 2.100 & 285 & 5 & $1,7 \%$ \\
\hline $8: 30$ & $19,0 \%$ & 190 & 2.290 & 279 & 6 & $2,0 \%$ \\
\hline $8: 35$ & $18,0 \%$ & 180 & 2.470 & 272 & 7 & $2,3 \%$ \\
\hline $8: 40$ & $17,0 \%$ & 170 & 2.640 & 264 & 8 & $2,7 \%$ \\
\hline $8: 45$ & $16,0 \%$ & 160 & 2.800 & 255 & 9 & $3,0 \%$ \\
\hline $8: 50$ & $15,0 \%$ & 150 & 2.950 & 245 & 10 & $3,3 \%$ \\
\hline $8: 55$ & $14,0 \%$ & 140 & 3.090 & 234 & 11 & $3,7 \%$ \\
\hline $9: 00$ & $13,0 \%$ & 130 & 3.220 & 222 & 12 & $4,0 \%$ \\
\hline $9: 05$ & $12,0 \%$ & 120 & 3.340 & 209 & 13 & $4,3 \%$ \\
\hline $9: 10$ & $11,0 \%$ & 110 & 3.450 & 195 & 14 & $4,7 \%$ \\
\hline $9: 15$ & $10,0 \%$ & 100 & 3.550 & 180 & 15 & $5,0 \%$ \\
\hline $9: 20$ & $9,0 \%$ & 90 & 3.640 & 164 & 16 & $5,3 \%$ \\
\hline $9: 25$ & $8,0 \%$ & 80 & 3.720 & 147 & 17 & $5,7 \%$ \\
\hline $9: 30$ & $7,0 \%$ & 70 & 3.790 & 129 & 18 & $6,0 \%$ \\
\hline $9: 35$ & $6,0 \%$ & 60 & 3.850 & 110 & 19 & $6,3 \%$ \\
\hline $9: 40$ & $5,0 \%$ & 50 & 3.900 & 90 & 20 & $6,7 \%$ \\
\hline $9: 45$ & $4,0 \%$ & 40 & 3.940 & 69 & 21 & $7,0 \%$ \\
\hline $9: 50$ & $3,0 \%$ & 30 & 3.970 & 47 & 22 & $7,3 \%$ \\
\hline $9: 55$ & $2,0 \%$ & 20 & 3.990 & 24 & 23 & $7,7 \%$ \\
\hline $10: 00$ & $1,0 \%$ & 10 & 4.000 & 0 & 24 & $8,0 \%$ \\
\hline$\sum$ & & 3.000 & & & 300 & \\
\hline
\end{tabular}

Fuente: Elaboración del autor

Además, resulta revelador anotar que entre los valores estadísticos de tendencia central que arrojó el escenario experimental, el parámetro de la mediana $M e$ correspondió exactamente al precio de $\$ 3.850$. Esta coincidencia se explicaría en el hecho de que una alta concentración de elecciones alrededor del punto de convergencia $E$, obviamente condicionaría los correspondientes valores de la mediana, moda y media.

Los resultados obtenidos sugieren que, en efecto, el tomador de decisión está en capacidad de percibir tal transición. $Y$ en consecuencia, el punto de convergencia $E$ representaría el valor, no en el cual, sino a partir del cual las probabilidades de un acuerdo son más altas y favorables. Esto significa que aunque la zona de precipitación de decisiones está identificada a la derecha del punto de convergencia $E$, no es posible anticipar el momento exacto en el que se producirá un acuerdo. Resulta prometedor, sin embargo, manifestar que tal zona de precipitación de decisiones está delimitada por dos marcadores que las partes los pueden identificar: el punto de convergencia $E$; y el límite máximo de expectativas. Esto se confirma con la evidencia obtenida por la prueba experimental explicada en el presente artículo. La proporción más significativa de decisiones de precio - tanto en términos de relevancia estadística experimental, como en cantidad de elecciones- se contabilizó en el área comprendida a partir de los $\$ 3.850$ en adelante, hasta el límite de $\$ 4.000$.

El modelo propuesto, aunque no acertó en la predicción puntual, resultó ser exacto en la demarcación de las zonas de baja y alta propensión a negociar, esto es, antes y después del punto de convergencia $E$. Puesto que este rango de precios fue notablemente superior al que prevaleció en el escenario de control, se infiere entonces que bajo las condiciones de 
Negociación Abierta las partes negociadoras pudieron alcanzar acuerdos de mayor valor agregado.

Este modelo puede encontrar aplicación práctica en el análisis y conducción de toda interacción social. Por una parte, los agentes económicos y sociales, interactúan llevados por su propio y legítimo interés de mejorar o fortalecer su situación de partida. Y por otra, esta dinámica siempre implica costos de transacción y de oportunidad para todas las partes intervinientes. En consecuencia, el marco conceptual y metodológico de la Negociación Abierta, es aplicable en todos los ámbitos de la interrelación humana, donde se requiera establecer compromisos o acuerdos para el cumplimiento de un objetivo: familia, comunidad, empresa, gobierno y países.

\section{Conclusiones}

La investigación realizada es concluyente respecto a la siguiente caracterización esencial de un proceso de Negociación Abierta: un proceso en el que desde su inicio, las partes revelan información acerca de sus expectativas y límites de aceptación. Tal información coadyuvará a alcanzar un mejor acuerdo entre las partes, en contraste con un proceso de negociación en el que exista incertidumbre respecto de las expectativas y límites de aceptación de las partes. Los resultados de la investigación sugieren la funcionalidad de un modelo predictivo, que se propone denominar Teorema de Negociación Abierta, y cuyo enunciado puede dictarse así: en un proceso de Negociación Abierta, las partes no llegarán a un acuerdo sino después de haber alcanzado el punto de intersección de sus costos de oportunidad, expresados como tasas de variación porcentual marginal.

Como corolario del referido teorema: dicho punto de intersección marcará el inicio de la zona de precipitación de decisiones donde las partes tendrán incentivos para llegar a un acuerdo que favorecerá sus intereses.

Por último, se sugiere que en subsiguientes investigaciones sobre Negociación Abierta, se estudie la correlación entre el género o zona geográfica, versus el perfil de decisiones o propensión a tomar riesgos. El alcance puede ser aún más amplio al relacionar variables culturales, psicosociales o laborales. 


\section{Referencias}

Bazerman, M. y Neale, M. (1992). Negotiating rationally. New York: Free Press. ISBN 0029019850.

Fisher, R., Ury, W., y Patton, B. (1979). Getting to YES: Negotiating Agreement Without Giving In. New York: Viking/Penguin.

Henao, C., Fierro, I., y Cardona, D.(2017). La negociación profesional, un acercamiento conceptual. Revista Espacios, 38 (32), 12.

Hyder, E.B., Prietula, M.J., y Weingart, L.R. (2000). Getting to best: Efficiency versus optimality in negotiation. Cognitive Science, 24(2), 169-204.

Lax, D. y Sebenius, J. (1986). The manager as negotiator: bargaining for cooperation and competitive gain. New York: Free Press. ISBN 0029187702.

Mehta, K. (2012). Five Essential Strategies for Creative Negotiations. IESE Insight, (15), 50-57.

Mullender, R. (2014). Negociación: el poder de la escucha activa. IESE Insight, (20), 67-71.

Olekalns, M., Smith, P.L., y Walsh, T. (1996). The process of negotiating: Strategies, timing, and outcomes. Organizational Behavior and Human Decisions Processes, 67, 61-77.

Olekalns, M. y Smith, P.L. (2000). Negotiating optimal outcomes: The role of strategic sequences in competitive negotiations. Human Communication Research, 24, 528-560.
Olekalns, M. y Smith, P. (2000). Understanding optimal outcomes: The role of strategy sequences in competitive negotiations. Human Communication Research, 26, 527-557.

Pruitt, D.G. (2005). Field Experiments on Social Conflict. International Negotiation, 10(1), 33-49.

Raiffa, H. (1982). The Art and Science of Negotiation. Harvard University. Press, Cambridge, MA.

Sebenius, J.K. (2001). Six Habits of Merely Effective Negotiators. Harvard Business Review, April.

Weingart, L. R., Olekalns, M., y Smith, P. L. (2004).Quantitative Coding of Negotiation Behavior. International Negotiation, 9(3), 441-455.

Weingart, L.R., Thompson, L.L., Bazerman, M.H., y Carroll, J.S. (1990). Tactical behavior and negotiation outcomes. The International Journal of Conflict Management, 1.

Weingart, L.R., Hyder, E.B., y Prietula, M.J. (1996). Knowledge matters: The effect of tactical descriptions on negotiation behavior and outcome. Journal of Personality and Social Psychology, 70, 1205-1217. 